\title{
Low preoperative serum prealbumin levels and the postoperative surgical site infection risk in elective spine surgery: a consecutive series
}

\author{
David J. Salvetti, MD, ${ }^{1}$ Zachary J. Tempel, MD, ${ }^{1}$ Ezequiel Goldschmidt, MD, PhD, ${ }^{1}$ \\ Nicole A. Colwell, MD, ${ }^{2}$ Federico Angriman, MD, MPH, ${ }^{3}$ David M. Panczykowski, MD, ${ }^{1}$ \\ Nitin Agarwal, MD, ${ }^{1}$ Adam S. Kanter, MD, ${ }^{1}$ and David O. Okonkwo, MD, PhD'1 \\ ${ }^{1}$ Department of Neurological Surgery, University of Pittsburgh Medical Center, Pittsburgh, Pennsylvania; ${ }^{2}$ Department of \\ Neurological Surgery, University of Utah, Salt Lake City, Utah; and 'Department of Medicine, Hospital Italiano de Buenos Aires, \\ Argentina
}

\begin{abstract}
OBJECTIVE Nutritional deficiency negatively affects outcomes in many health conditions. In spine surgery, evidence linking preoperative nutritional deficiency to postoperative surgical site infection (SSI) has been limited to small retrospective studies. Authors of the current study analyzed a large consecutive cohort of patients who had undergone elective spine surgery to determine the relationship between a serum biomarker of nutritional status (preoperative prealbumin levels) and SSI.
\end{abstract}

METHODS The authors conducted a retrospective review of the electronic medical charts of patients who had undergone posterior spinal surgeries and whose preoperative prealbumin level was available. Additional data pertinent to the risk of SSI were also collected. Patients who developed a postoperative SSI were identified, and risk factors for postoperative SSI were analyzed. Nutritional deficiency was defined as a preoperative serum prealbumin level $\leq 20 \mathrm{mg} / \mathrm{dl}$.

RESULTS Among a consecutive series of 387 patients who met the study criteria for inclusion, the infection rate for those with preoperative prealbumin $\leq 20 \mathrm{mg} / \mathrm{dl}$ was $17.8 \%$ (13/73), versus $4.8 \%$ (15/314) for those with preoperative prealbumin $>20 \mathrm{mg} / \mathrm{dl}$. On univariate and multivariate analysis a low preoperative prealbumin level was a risk factor for postoperative SSI with a crude OR of $4.29(p<0.01)$ and an adjusted OR of $3.28(p=0.02)$. In addition, several previously known risk factors for infection, including diabetes, spinal fusion, and number of operative levels, were significant for the development of an SSI.

CONCLUSIONS In this consecutive series, preoperative prealbumin levels, a serum biomarker of nutritional status, correlated with the risk of SSI in elective spine surgery. Prehabilitation before spine surgery, including strategies to improve nutritional status in patients with nutritional deficiencies, may increase value and improve spine care.

https://thejns.org/doi/abs/10.3171/2018.3.SPINE171183

KEYWORDS prealbumin; surgical site infections; spine surgery; nutritional deficiency; infection risk

$\mathrm{S}$ URGICAL site infection (SSI) after elective spine surgery represents a potentially preventable complication that portends significant cost to the healthcare system and serious morbidity for patients. ${ }^{7,12}$ A potentially modifiable risk factor for the development of an SSI is a patient's nutritional status, and across other specialties, serum biomarkers of nutritional status have been shown to be closely linked to clinical outcomes and complications. ${ }^{1,8,13,18}$ The classically used surrogate biomarker for the measurement of nutritional status is serum albumin, and previous studies have identified an association between SSI after elective spine surgery and low preopera- tive albumin levels..$^{10,15,16}$ However, hypoalbuminemia is a relatively nonspecific finding caused by a number of different disease processes, and debate exists on how well it reflects malnutrition., ${ }^{2,36}$ The currently preferred serum biomarker of nutritional status is prealbumin, or transthyretin. Prealbumin is largely composed of essential amino acids, making its production highly dependent on adequate nutritional intake, and it has a shorter half-life than albumin. With a higher protein turnover, it is believed that prealbumin levels provide a more sensitive estimation of nutritional status than albumin.

Low prealbumin levels have also been shown to be a

ABBREVIATIONS BMI = body mass index; $\mathrm{SSI}=$ surgical site infection .

SUBMITTED October 30, 2017. ACCEPTED March 29, 2018.

INCLUDE WHEN CITING Published online July 27, 2018; DOI: 10.3171/2018.3.SPINE171183. 
predictor of SSI both preoperatively and at the time of re-presentation with a wound infection..$^{14,17}$ Despite this increasing evidence that malnutrition may influence the risk of infection, no practical strategies or guidelines have been proposed for the management of preoperative nutritional deficiency in spine surgery. Moreover, studies have been limited by small sample sizes. In the current study we analyze a large consecutive cohort of patients who underwent elective spine surgery in order to determine the relationship between preoperative prealbumin and SSI.

\section{Methods \\ Data Collection}

In 2016, we conducted a retrospective review of the electronic medical charts of consecutive patients who had undergone elective spinal surgery performed by the two senior authors between October 2009 and December 2015. This study was conducted under an institutional review board-approved protocol at the University of Pittsburgh that did not require individual consent. Included patients were those who had undergone open posterior decompressions and/or fusions for spondylosis at all levels throughout the spine. Patients who had undergone anterior cervical fusion, lateral access surgery, and microdiscectomy were excluded since they have a theoretically minimal risk of infection. Patients who had undergone surgery in association with a malignancy were also excluded. Additionally, patients with adult spinal deformity were excluded given that this population has a unique risk profile for SSIs and is complicated by a diversity of surgical techniques to address spinal deformities.

Patients were excluded from our analysis if they did not have a preoperative prealbumin level available for review. The medical records for each patient were queried for age, diabetes, body mass index (BMI), smoking status, chronic steroid use, operative time, fusion versus decompression, and number of levels operated on. We then cross-referenced this list with department billing records for wound debridement, as well as with internal Department of Infectious Diseases records for patients meeting the Centers for Disease Control and Prevention (CDC) criteria for SSI, to identify all patients who developed a deep infection requiring wound debridement. On the basis of our retrospective work evaluating the relationship between prealbumin and SSI, we implemented a protocol to include prealbumin in the routine lab work obtained prior to elective spine surgery.

\section{Statistical Analysis}

Quantitative variables are presented as the mean and standard deviation or, in the case of noticeably skewed data, as the median and interquartile range. Baseline differences between patients with either normal or low prealbumin levels were assessed using the chi-square test for categorical variables and the Student t-test or Wilcoxon test for continuous variables, as appropriate. Finally, in order to estimate the association between prealbumin levels and SSI, we used univariate and multivariate logistic regression models. Nutritional deficiency was defined as a preoperative serum prealbumin level $\leq 20 \mathrm{mg} / \mathrm{dl}$. Co-
TABLE 1. Baseline characteristics of patients

\begin{tabular}{lccr}
\hline \multicolumn{1}{c}{ Parameter } & $\begin{array}{c}\text { Prealbumin } \\
\leq 20 \mathrm{mg} / \mathrm{dl}\end{array}$ & $\begin{array}{c}\text { Prealbumin } \\
>20 \mathrm{mg} / \mathrm{dl}\end{array}$ & $\begin{array}{c}\mathrm{p} \\
\text { Value }\end{array}$ \\
\hline No. of patients & 73 & 314 & \\
\hline Mean age in yrs (SD) & $59.0(12.5)$ & $54.9(15.4)$ & 0.02 \\
\hline Mean prealbumin level in & $16.9(3.47)$ & $29.3(6.3)$ & $<0.01$ \\
mg/dl (SD) & & & \\
\hline Male sex, no. (\%) & $36(50)$ & $148(47.6)$ & 0.14 \\
\hline Mean BMl in kg/m² (SD) & $28.5(6.7)$ & $30.0(6.5)$ & 0.10 \\
\hline Mean surgery time in mins (SD) & $226.2(85.3)$ & $212.0(89.0)$ & 0.21 \\
\hline Smoking status, no. (\%) & $18(24.7)$ & $70(22.3)$ & 0.66 \\
\hline Mean albumin level in g/dl (SD) & $3.72(2.22)$ & $3.89(0.66)$ & 0.54 \\
\hline Diabetes, no. (\%) & $7(9.6)$ & $61(19.4)$ & 0.05 \\
\hline Steroid treatment, no. (\%) & $5(6.9)$ & $32(10.2)$ & 0.38 \\
\hline Surgery type, no. (\%) & & & 0.79 \\
Lumbar fusion & $58(79.5)$ & $245(78.0)$ & \\
Lumbar laminectomy & $4(5.5)$ & $24(7.6)$ & \\
Thoracic fusion & $2(2.7)$ & $8(2.6)$ & \\
Thoracic laminectomy & $0(0)$ & $3(1)$ & \\
Cervical fusion & $8(11.0)$ & $33(10.5)$ & \\
Cervical laminectomy & $1(1.4)$ & $1(0.32)$ & \\
\hline Median no. of instrumented & $2(2)$ & $2(2)$ & 0.20 \\
levels (IQR) & & & \\
\hline
\end{tabular}

$\mathrm{SD}=$ standard deviation.

Means were compared with the use of t-tests for unequal variances, medians were compared with the Wilcoxon test, and proportions were compared with the use of the chi-square test.

variates considered for this model were those that had been deemed clinically relevant in addition to those that were significant in the bivariate analysis at the 0.2 level. The lineal assumption for continuous included covariates was tested by means of cubic restricted splines. A priori interactions were also tested. Selection of the final multivariate model was based on both likelihood ratio tests and Akaike's information criterion. Finally, we used receiver operating characteristic (ROC) curves to evaluate model discrimination and the Hosmer-Lemeshow statistic for the assessment of calibration. We used Stata version 14.2 for all analyses (StataCorp LP).

\section{Results}

Three hundred eighty-seven patients met the study inclusion criteria. Preoperative prealbumin values ranged from 7 to $49 \mathrm{mg} / \mathrm{dl}$ with a mean of $28 \mathrm{mg} / \mathrm{dl}$. Overall, 73 (19\%) patients had a prealbumin level $\leq 20 \mathrm{mg} / \mathrm{dl}$. Table 1 details the characteristics of the patient cohort broken into those with normal and those with low prealbumin. A total of 28 infections (7.2\%) were identified in the entire cohort. The infection rate for patients with preoperative prealbu$\min \leq 20 \mathrm{mg} / \mathrm{dl}$ was $17.8 \%$ (13/73), versus $4.7 \%$ (15/314) for the patients with preoperative prealbumin $>20 \mathrm{mg} / \mathrm{dl}$. Patients with low prealbumin levels were significantly older $(59.0 \pm 12.5$ vs $54.9 \pm 15.4$ years, $\mathrm{p}=0.02)$ and had a lower incidence of diabetes (7 [9.6\%] vs 61 [19.4\%]). The BMI tended to be larger in patients with normal prealbumin 
TABLE 2. Crude and adjusted odds ratios of SSI for prealbumin and other potential risk factors

\begin{tabular}{|c|c|c|c|c|}
\hline Factor & Crude OR $(95 \% \mathrm{Cl})$ & p Value* & Adjusted OR $(95 \% \mathrm{Cl})$ & $p$ Value \\
\hline Prealbumin $\leq 20 \mathrm{mg} / \mathrm{dl}$ & $4.29(1.94-9.48)$ & $<0.01$ & $3.28(1.19-9.09)$ & 0.02 \\
\hline Age & $1.04(1.01-1.07)$ & 0.01 & $1.02(0.98-1.06)$ & 0.43 \\
\hline BMI & $1.02(0.96-1.09)$ & 0.47 & $1.01(0.94-1.09)$ & 0.72 \\
\hline Duration of surgery & $1.02(0.79-1.33)$ & 0.85 & & \\
\hline Current smoker & $0.91(0.36-2.33)$ & 0.85 & & \\
\hline Albumin & $0.63(0.41-0.99)$ & 0.04 & $0.72(0.41-1.27)$ & 0.26 \\
\hline Diabetes & $1.73(0.70-4.28)$ & 0.23 & $3.30(1.03-10.62)$ & 0.05 \\
\hline Steroid use & $1.23(0.36-4.29)$ & 0.75 & & \\
\hline Male sex & $0.62(0.27-1.38)$ & 0.24 & & \\
\hline Spinal fusion & $0.28(0.09-0.91)$ & 0.03 & $0.13(0.04-0.51)$ & $<0.01$ \\
\hline Instrumented levels & $1.10(1.00-1.21)$ & 0.05 & $1.14(1.02-1.28)$ & 0.02 \\
\hline
\end{tabular}

levels $\left(30.0 \pm 6.5 \mathrm{~kg} / \mathrm{m}^{2}\right.$ vs $\left.28.5 \pm 6.7 \mathrm{~kg} / \mathrm{m}^{2}\right)$, but the difference did not reach statistical significance. Both groups were similar in terms of patient sex, smoking status, steroid treatment, type or length of surgery, and number of instrumented levels. Table 2 details the results of a univariate and multivariate logistic regression to assess the association of different variables with the development of an SSI. On univariate logistic regression the crude OR for having an SSI in the low prealbumin group was 4.29 (95\% CI 1.94-9.48); in the multivariate model the adjusted OR was 3.28 (95\% CI 1.19-9.09). Age and albumin were significant on univariate models, but when adjusted for other variables on multivariate analysis, they did not demonstrate statistical significance. Diabetes, fusion versus decompression, and number of instrumented levels were all statistically significant as risk factors for infection in the multivariate model.

\section{Discussion}

Nutritional deficiency, as defined by low preoperative prealbumin levels, was a major risk factor for SSI in this consecutive cohort of elective spine surgery patients. These findings reinforce and strengthen our prior retrospective findings that low prealbumin levels are associated with SSIs. ${ }^{14,17}$ In an initial retrospective cohort study by Tempel et al., 83 patients with postoperative infections requiring surgical debridement were identified, and 82/83 (99\%) patients were found to have abnormally low prealbumin levels at the time the postoperative infection was diagnosed. ${ }^{17}$ While this was an important observation and has clinical utility in identifying patients who have infections, the causality of whether low prealbumin levels preoperatively portend an increased risk could not be established, especially considering the known relationship of decreased prealbumin levels as part of an acute phase response. Subsequently, our group performed a case control study involving 32 patients who developed an SSI compared to 72 controls who did not. ${ }^{14} \mathrm{~A}$ preoperative prealbumin level $<20 \mathrm{mg} / \mathrm{dl}$ portended an HR of 2.12 for the risk of developing an SSI. This study was limited in terms of its sample size, which we aimed to address with the current study.
The finding herein of an adjusted OR of 3.28 indicates a strong association between a low preoperative prealbumin level and the risk of an SSI in elective spine surgery.

This study also demonstrated a significant relationship between factors previously associated with infection, including diabetes, fusion versus decompression, and number of levels fused. ${ }^{15,16}$ This fact reinforces the need for a thorough medical evaluation prior to operative intervention to optimize a patient's medical comorbidities such as diabetes-i.e., prehabilitation. Other factors expected to have an association with infection such as steroid use and smoking proved to be statistically insignificant. This finding could reflect limitations in the retrospective nature of our review and the study's primary design in analyzing prealbumin's prognostic utility. For instance, information on pack-years of smoking history was inconsistently available; therefore, we chose to collect these data as a categorical statistic of yes/no as opposed to a continuous variable. Similarly, steroid use was collected categorically. This limitation may influence the ability to delineate these factors as infection risks.

With increasing evidence that prealbumin levels predict the postoperative SSI risk, the ultimate goal is to utilize this information to lower infection risk and improve patient care. After a patient with a low prealbumin level is identified, nutritional supplementation could be implemented prior to surgery. To date, there has been minimal work in regard to nutritional supplementation in spine surgery, and the trials that have been performed have largely had negative to negligible results. ${ }^{9,11}$ As regards nutritional supplementation, there is substantial evidence that regimens containing protein supplementation, antioxidants, and calcium can promote wound healing, particularly in chronic wounds such as sacral decubitus ulcers. ${ }^{4}$ Therefore, it seems reasonable that such a regimen would have utility in the perioperative setting to promote postoperative healing. At our institute, we utilize a regimen of protein-enriched milkshakes (twice a day), vitamin C (500 mg daily), vitamin D (400 IU daily), calcium carbonate (1500 $\mathrm{mg}$ thrice a day), and zinc gluconate (50 $\mathrm{mg}$ daily) in patients actively treated for an SSI. Further study is need- 
ed to elucidate whether the preoperative administration of such a regimen can alter the risk profile of at-risk patients.

The current analysis aims to overcome limitations of prior evaluations of prealbumin by analyzing a large consecutive cohort with preoperative prealbumin levels. Nonetheless, our study has limitations in that the patient cohort was derived from the experience of only two surgeons and the risk factor data were collected retrospectively. Moreover, although hepatic proteins such as prealbumin and albumin have long been utilized as biomarkers of nutritional status, adequate nutrition is a complex state. The supposition that a single biomarker can assess such a complex state is debatable. The serum levels of both albumin and prealbumin are susceptible to influence from general inflammatory states, and prealbumin levels are known to be decreased during the acute phase response. ${ }^{5}$ This debate does not negate prealbumin's utility as a prognostic tool for identifying patients at risk for an SSI, but it may influence our ability to alter that risk profile. Despite some limitations, this study represents strong evidence that low preoperative prealbumin levels increase the risk of an SSI in elective spine surgery patients.

\section{Conclusions}

A low preoperative serum prealbumin level was associated with a higher risk of developing an SSI after elective spine surgery. Preoperative assessment of nutritional status can inform prehabilitation options to reduce the risk of SSI. Further work is required to determine whether such a strategy could result in a substantive decrease in SSIs.

\section{References}

1. Badjatia N, Monahan A, Carpenter A, Zimmerman J, Schmidt JM, Claassen J, et al: Inflammation, negative nitrogen balance, and outcome after aneurysmal subarachnoid hemorrhage. Neurology 84:680-687, 2015

2. Beck FK, Rosenthal TC: Prealbumin: a marker for nutritional evaluation. Am Fam Physician 65:1575-1578, 2002

3. Bernstein LH, Ingenbleek Y: Transthyretin: its response to malnutrition and stress injury. Clinical usefulness and economic implications. Clin Chem Lab Med 40:1344-1348, 2002

4. Cereda E, Klersy C, Serioli M, Crespi A, D’Andrea F: A nutritional formula enriched with arginine, zinc, and antioxidants for the healing of pressure ulcers: a randomized trial. Ann Intern Med 162:167-174, 2015

5. Fuhrman MP, Charney P, Mueller CM: Hepatic proteins and nutrition assessment. J Am Diet Assoc 104:1258-1264, 2004

6. Gatta A, Verardo A, Bolognesi M: Hypoalbuminemia. Intern Emerg Med 7 (Suppl 3):S193-S199, 2012

7. Goldschmidt E, Rasmussen J, Chabot JD, Gandhoke G, Luzzi E, Merlotti L, et al: The effect of vancomycin powder on human dural fibroblast culture and its implications for dural repair during spine surgery. J Neurosurg Spine 25:665-670, 2016

8. Harriman S, Rodych N, Hayes P, Moser MA: The C-reactive protein-to-prealbumin ratio predicts fistula closure. Am J Surg 202:175-178, 2011

9. Hu SS, Fontaine F, Kelly B, Bradford DS: Nutritional depletion in staged spinal reconstructive surgery. The effect of total parenteral nutrition. Spine (Phila Pa 1976) 23:14011405,1998

10. Klein JD, Hey LA, Yu CS, Klein BB, Coufal FJ, Young EP, et al: Perioperative nutrition and postoperative complications in patients undergoing spinal surgery. Spine (Phila Pa 1976) 21:2676-2682, 1996

11. Lapp MA, Bridwell KH, Lenke LG, Baldus C, Blanke K, Iffrig TM: Prospective randomization of parenteral hyperalimentation for long fusions with spinal deformity: its effect on complications and recovery from postoperative malnutrition. Spine (Phila Pa 1976) 26:809-817, 2001

12. Lieber B, Han B, Strom RG, Mullin J, Frempong-Boadu AK, Agarwal N, et al: Preoperative predictors of spinal infection within the National Surgical Quality Inpatient database. World Neurosurg 89:517-524, 2016

13. Pinilla JC, Hayes P, Laverty W, Arnold C, Laxdal V: The Creactive protein to prealbumin ratio correlates with the severity of multiple organ dysfunction. Surgery 124:799-806, 1998

14. Salvetti DJ, Tempel ZJ, Gandhoke GS, Parry PV, Grandhi RM, Kanter AS, et al: Preoperative prealbumin level as a risk factor for surgical site infection following elective spine surgery. Surg Neurol Int 6 (Suppl 19):S500-S503, 2015

15. Schoenfeld AJ, Carey PA, Cleveland AW III, Bader JO, Bono $\mathrm{CM}$ : Patient factors, comorbidities, and surgical characteristics that increase mortality and complication risk after spinal arthrodesis: a prognostic study based on 5,887 patients. Spine J 13:1171-1179, 2013

16. Schoenfeld AJ, Ochoa LM, Bader JO, Belmont PJ Jr: Risk factors for immediate postoperative complications and mortality following spine surgery: a study of 3475 patients from the National Surgical Quality Improvement Program. J Bone Joint Surg Am 93:1577-1582, 2011

17. Tempel Z, Grandhi R, Maserati M, Panczykowski D, Ochoa $\mathrm{J}$, Russavage J, et al: Prealbumin as a serum biomarker of impaired perioperative nutritional status and risk for surgical site infection after spine surgery. J Neurol Surg A Cent Eur Neurosurg 76:139-143, 2015

18. Xie Q, Zhou Y, Xu Z, Yang Y, Kuang D, You H, et al: The ratio of CRP to prealbumin levels predict mortality in patients with hospital-acquired acute kidney injury. BMC Nephrol 12:30, 2011

\section{Disclosures}

The authors report no conflict of interest concerning the materials or methods used in this study or the findings specified in this paper.

\section{Author Contributions}

Conception and design: Salvetti, Tempel, Kanter, Okonkwo. Acquisition of data: Salvetti, Tempel, Angriman, Panczykowski. Analysis and interpretation of data: Salvetti, Tempel, Goldschmidt, Colwell, Angriman, Panczykowski. Drafting the article: Salvetti, Tempel, Colwell. Critically revising the article: Goldschmidt, Angriman, Panczykowski, Agarwal, Kanter, Okonkwo. Reviewed submitted version of manuscript: all authors. Statistical analysis: Goldschmidt, Angriman, Panczykowsk. Administrative/ technical/material support: Kanter, Okonkwo. Study supervision: Kanter, Okonkwo.

\section{Supplemental Information \\ Previous Presentations}

Data that pertains to this paper were presented in preliminary form as an oral presentation at the 2016 CNS Spine Summit held in Orlando, Florida, on March 18, 2016. Moreover, preliminary data were published in the UPMC neurosurgery department newsletter.

\section{Correspondence}

David J. Salvetti: University of Pittsburgh Medical Center, Pittsburgh,PA. salvettidj@upmc.edu. 